\title{
Chọn lựa nút chuyển tiếp nâng cao hiệu năng mạng vô tuyến nhận thức dạng nền với sự xuất hiện của nút nghe lén và khiếm khuyết phần cứng Performance Enhancement of Underlay Cognitive Radio Networks with Relay Selection Methods under Presence of Eavesdropper and Hardware Impairments
}

\author{
Phạm Thị Đan Ngọc, Trần Trung Duy, Võ Nguyễn Quốc Bảo, Hồ Văn Khương
}

\begin{abstract}
In this paper, we study physical-layer security issue of secondary networks in cognitive radio (CR). In the considered system model, a secondary source communicates with a secondary destination with assistance of multiple secondary relays in presence of multiple secondary eavesdroppers. The secondary users operate on an underlay mode, where they must adjust their transmit power to satisfy interference constraints required by primary users. Moreover, we propose three efficient relay selection methods to improve outage performance for the data links as well as to reduce decoding probability (DP) of the eavesdropping links. For performance evaluation and comparison, we derive exact closed-form expressions of outage probability $(O P)$ and decoding probability $(D P)$ over Rayleigh fading channel under impact of imperfect hardware transceiver. Finally, Monte Carlo simulations are performed to verify our theoretical derivations. The results present that with the presence of the eavesdroppers, there always exists a trade-off between security and reliability.
\end{abstract}

Keywords: Underlay cognitive radio, physicallayer security, hardware impairments, relay selection, Rayleigh fading channel, outage probability, decoding probability.

\section{GIỚI THIỆ}

Vô tuyến nhận thức (Cognitive Radio) được đề xuất bởi Joseph Mitola, là một giải pháp hiệu quả, nhằm giải quyết vấn đề khan hiếm phổ tần trong các mạng truyền thông vô tuyến [1]. Trong vô tuyến nhận thức, mạng sơ cấp (Primary network) được cấp phép sử dụng phổ tần, trong khi mạng thứ cấp (Secondary network) chỉ có thể sử dụng các băng tần trống (các băng tần đang không được sử dụng bởi mạng sơ cấp).

Thông thường, những người dùng thứ cấp (Secondary users) phải thăm dò phổ [2], [3] để tìm ra các băng tần trống và sử dụng chúng. Tuy nhiên, khi những người dùng sơ cấp (Primary users) bắt đầu sử dụng các băng tần này, các người dùng thứ cấp phải ngay lập tức tìm kiếm các phổ tần trống khác để truy nhập. Hệ quả là sự truyền dữ liệu của mạng thứ cấp sẽ không được liên tục và hiệu năng của mạng cũng phụ thuộc hoàn toàn vào sự xuất hiện của người dùng so cấp. Hơn thế nữa, việc thăm dò phổ có thể không chính xác, gây nên các hoạt động cảnh báo sai lầm (miss detection và false alarm) [2], [3] làm ảnh hưởng nghiêm trọng đến chất lượng dịch vụ (Quality of service (QoS)) của cả hai hệ thống.

Gần đây, các nhà nghiên cứu đề xuất một kỹ thuật vô tuyến nhận thức hiệu quả, với tên gọi vô tuyến nhận thức dạng nền (Underlay cognitive radio) [4], [5], [6], nhằm đảm bảo tính liên tục truyền/nhận cho 
mạng thứ cấp. Trong kỹ thuật này, hai mạng sơ cấp và thứ cấp có thể cùng lúc sử dụng phổ tần số. Tuy nhiên, người dùng thứ cấp phải sử dụng mức công suất phát đủ thấp để giao thoa gây lên trên mạng sơ cấp không ảnh hưởng đến chất lượng dịch vụ của mạng này [4], [5], [6]. Với công suất phát giới hạn, hiệu năng của mạng thứ cấp bị suy giảm trầm trọng, đặc biệt trong môi trường kênh fading Rayleigh. Để đạt được hiệu năng cao hơn, các nhà nghiên cứu đã sử dụng kỹ thuật chuyển tiếp cho mạng này. Các kết quả trong [4], [5], [6] cho thấy rằng các giao thức chuyển tiếp phân tập nâng cao độ lợi phân tập và giảm tốc độ lỗi cho mạng người dùng thứ cấp.

Trong khi các nhà nghiên cứu đang nỗ lực tìm ra các giải pháp nhằm cải thiện hiệu năng của mạng thứ cấp, thì việc bảo mật thông tin cho mạng này vẫn chưa nhận được sự quan tâm đúng mức. Bởi tính chất quảng bá của kênh truyền vô tuyến, những người dùng không hợp pháp có thể dễ dàng nghe trộm thông tin được phát đi trong mạng. Cho đến nay, những thuật toán bảo mật phổ biến như Data Encryption Standard (DES), Advanced Encryption Standard (AES), RSA, v.v. đều là các kỹ thuật khá phức tạp, và có thể khó khả thi khi triển khai trên các thiết bị sử dụng trong mạng thứ cấp.

Gần đây, bảo mật thông tin lớp vật lý (physicallayer security) [7], [8] đã được phát triển nhằm đạt được hiệu quả bảo mật, trong khi giảm thiểu đáng kể sự phức tạp trong quá trình hiện thực. Thật vậy, sự bảo mật này có thể đạt được dựa vào các tính chất vật lý của kênh truyền như khoảng cách, thông tin trạng thái kênh truyền (Channel state information) hay bằng việc tạo nhiễu nhân tạo (Artifial noise) lên các thiết bị nghe lén. Một lần nữa, chuyển tiếp phân tập lại trở thành một giải pháp hiệu quả nâng cao hiệu năng bảo mật lớp vật lý cho các hệ thống truyền thông vô tuyến.

Trong tài liệu tham khảo [9], nhóm các tác giả đề xuất mô hình chọn nút chuyển tiếp cho mạng chuyển tiếp cộng tác, trong đó nút chuyển tiếp tốt nhất là nút đạt được dung lượng bảo mật cực đại. Các tác giả trong công trình [10] khảo sát vấn đề bảo mật thông tin lớp vật lý cho các hệ thống khuếch đại và chuyển tiếp với các nút chuyển tiếp không tin cậy.

Các công trình [11], [12] nghiên cứu hiệu năng bảo mật của mạng thứ cấp trong môi trường vô tuyến nhận thức dạng nền trên kênh truyền fading Rayleigh, thông qua các thông số hiệu năng như: dung lượng bảo mật trung bình (Average secrecy capacity), xác suất dừng bảo mật (Secrecy outage probability) và xác suất dung lượng bảo mật khác không (Probability of non-zero secrecy capacity). Cũng vậy, các mô hình trong [11], [12] cải thiện đáng kể hiệu quả bảo mật nhờ vào các phương pháp chọn lựa nút chuyển tiếp và nút tạo nhiễu (jammer).

Tuy nhiên, hầu hết các nghiên cứu về bảo mật lớp vật lý không quan tâm đến khả năng giải mã tín hiệu của nút nghe lén. Thật vậy, một khi nút nghe lén có thể giải mã thành công dữ liệu nghe trộm thì sự bảo mật là không còn nữa. Trong công trình [13] các tác giả nghiên cứu khả năng giải mã dữ liệu tại nút nghe lén và xác suất dừng tại nút đích trong mạng chuyển tiếp thứ cấp. Các kết quả trong [13] cho thấy rằng có một sự đánh đổi giữa khả năng bảo mật thông tin và xác suất dừng của hệ thống.

Tuy nhiên, các tác giả trong các công trình [11], [12], [13] đều giả sử rằng phần cứng của các bộ thu/phát thứ cấp là lý tưởng. Tuy nhiên, trong thực tế, phần cứng của các thiết bị này là không lý tưởng, xuất phát từ sự không tuyến tính của bộ khuếch đại, sự nhiễu pha hay sự mất cân bằng $\mathrm{I} / \mathrm{Q}, \mathrm{v} . \mathrm{v}$... Sự khiếm khuyết phần cứng sẽ làm giảm đáng kể về hiệu năng của các hệ thống truyền thông vô tuyến [14], [15]. Trong tài liệu tham khảo [16], các tác giả lần đầu tiên đánh giá chính xác xác suất dung lượng bảo mật khác 0 trong chuyển tiếp đa chặng. Tuy nhiên, mô hình này chỉ khảo sát sự truyền dữ liệu trực tiếp (Direct transmission), mà không đưa ra các phương pháp chọn lựa nút chuyển tiếp để nâng cao hiệu năng bảo mật của hệ thống. 
Trong bài báo này, chúng tôi sẽ nghiên cứu sự ảnh hưởng của phần cứng không lý tưởng lên hiệu năng của mạng thứ cấp trong vô tuyến nhận thức dạng nền, thông qua đại lượng xác suất dừng $(\mathrm{OP})$ tại nút đích thứ cấp và khả năng giải mã DP (Decoding Probability) của nút nghe lén thứ cấp ${ }^{1}$. Các kết quả trong bài báo này được phát triển từ công trình [17] của chúng tôi. Tuy nhiên, khác với [17], chúng tôi xem xét mô hình tổng quát với sự xuất hiện của nhiều người nghe lén thứ cấp.

Bài báo đề xuất ba phương pháp lựa chọn nút chuyển tiếp thứ cấp nhằm giảm giá trị $\mathrm{OP}$ cho hệ thống, đồng thời cũng giảm chất lượng kênh truyền đến nút nghe lén. Trong phương pháp thứ nhất (được đặt tên là HCG-D (Highest Channel Gain to Destination)), nút chuyển tiếp có độ lợi kênh truyền đến nút đích lớn nhất sẽ được chọn để chuyển tiếp dữ liệu. Trong phương pháp thứ hai (với tên gọi MCG-E (Minimum Channel Gain to Eavesdroppers)), hệ thống sẽ chọn nút chuyển tiếp, tương ứng với độ lợi kênh truyền nhỏ nhất đến các nút nghe lén. Cuối cùng, đề xuất thứ ba mang tên COMB (Combine) là mô hình kết hợp giữa hai đề xuất trước đó, nhằm tận dụng các ưu điểm và khắc phục các nhược điểm của $\mathrm{HCG}-\mathrm{D}$ và MCG-E.

Hơn thế nữa, chúng tôi đưa ra các công thức dạng đóng chính xác (Exact closed-form expression) cho các đại lượng $\mathrm{OP}$ và $\mathrm{DP}$ trên kênh truyền fading Rayleigh, trong sự xuất hiện của nhiều nút nghe lén thứ cấp và nhiều nút sơ cấp. Kế tiếp, chúng tôi thực hiện những mô phỏng máy tính, sử dụng phương pháp Monte Carlo, để kiểm tra độ chính xác của các biểu thức toán học. Các kết quả cho thấy rằng mô hình COMB đạt được hiệu năng ở giữa hai mô hình còn lại; và các thông số như mức suy hao phần cứng, số lượng

${ }^{1}$ Trong tài liệu [13], các tác giả sử dụng khái niệm xác suất chặn (Intercept probability) thay cho khái niệm khả năng giải mã (DP) như trong công trình này. nút sơ cấp, số nút nghe lén và ngưỡng giao thoa định mức ảnh hưởng đáng kể lên các giá trị $\mathrm{OP}$ và $\mathrm{DP}$.

Phần còn lại của bài báo được trình bày như sau. Mô hình hệ thống và các giao thức đề xuất được giới thiệu trong Phần II. Phần III phân tích và đánh giá hiệu năng của các phương pháp đề xuất. Kết quả mô phỏng và lý thuyết sẽ được thể hiện trong phần IV. Cuối cùng, các biện luận và hướng phát triển của nghiên cứu này sẽ được trình bày trong Phần $\mathrm{V}$.

\section{MÔ HÌNH HÊ THỐNG}

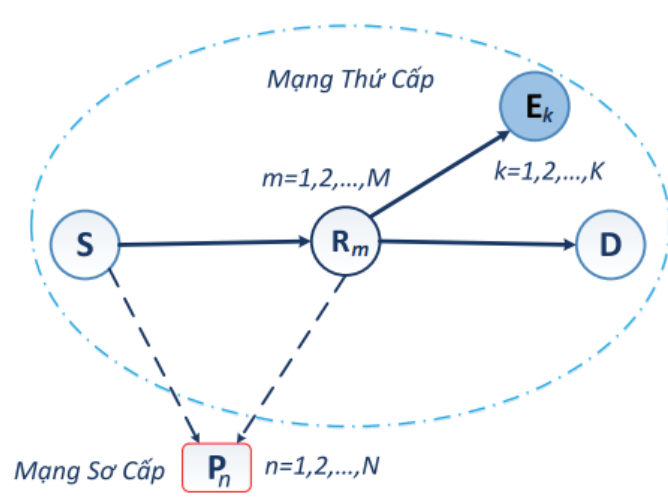

Hình 1. Mô hình hệ thống nghiên cứu

Hình 1 mô tả mô hình hệ thống được khảo sát trong bài báo. Trong mạng thứ cấp, nút nguồn thứ cấp (S) muốn gửi dữ liệu đến nút đích thứ cấp $(\mathrm{D})$, thông qua sự giúp đỡ của $M$ nút chuyển tiếp $\left(\mathrm{R}_{1}, \mathrm{R}_{2}, \ldots, \mathrm{R}_{M}\right)$. Giả sử rằng, nút nguồn $\mathrm{S}$ không có đường liên kết trực tiếp đến nút đích $\mathrm{D}$ bởi khoảng cách xa hay hiệu ứng fading che khuất. Do đó, các nút chuyển tiếp được sử dụng để đưa dữ liệu từ nguồn đến đích. Ngoài ra, trong mạng thứ cấp còn xuất hiện $K$ nút nghe lén thứ cấp được ký hiệu là $\mathrm{E}_{1}, \mathrm{E}_{2}, \ldots, \mathrm{E}_{K}$. Các nút này cố gắng nghe trộm dữ liệu được gửi đi từ các nút chuyển tiếp. Ta cũng giả sử rằng, các nút nghe lén nằm gần nút đích $\mathrm{D}$, và vì thế các nút này cũng không thể nhận dữ liệu được truyền từ nút nguồn. Cũng trong hình vẽ này, hệ thống sơ cấp gồm có $N$ người sơ cấp được đặt tên là $\mathrm{P}_{1}, \mathrm{P}_{2}, \ldots, \mathrm{P}_{N}$.

Ta ký hiệu $h_{\mathrm{SR}_{m}}, h_{\mathrm{R}_{m} \mathrm{D}}, h_{\mathrm{SP}_{n}}, h_{\mathrm{R}_{m} \mathrm{P}_{n}}$ và $h_{\mathrm{R}_{m} \mathrm{E}_{k}}$ lần lượt là hệ số kênh truyền fading Rayleigh của các liên kết 
$\mathrm{S} \rightarrow \mathrm{R}_{m}, \mathrm{R}_{m} \rightarrow \mathrm{D}, \mathrm{S} \rightarrow \mathrm{P}_{n}, \mathrm{R}_{m} \rightarrow \mathrm{P}_{n}$ và $\mathrm{R}_{m} \rightarrow \mathrm{E}_{k}$, với $m=1,2, \ldots, M, n=1,2, \ldots, N$ và $k=1,2, \ldots, K$. Như đã đề cập trong các tài liệu [4], [5], các độ lợi kênh truyền như $\quad \gamma_{\mathrm{SR}_{m}}=\left|h_{\mathrm{SR}_{m}}\right|^{2}, \gamma_{\mathrm{R}_{m} \mathrm{D}}=\left|h_{\mathrm{R}_{m} \mathrm{D}}\right|^{2}$, $\gamma_{\mathrm{SP}_{n}}=\left|h_{\mathrm{SP}_{n}}\right|^{2}, \gamma_{\mathrm{R}_{m} \mathrm{P}_{n}}=\left|h_{\mathrm{R}_{m} \mathrm{P}_{n}}\right|^{2}$ và $\gamma_{\mathrm{R}_{m} \mathrm{E}_{k}}=\left|h_{\mathrm{R}_{m} \mathrm{E}_{k}}\right|^{2}$ sẽ là các biến ngẫu nhiên có phân phối mũ (Exponential random variable). Cụ thể, hàm phân phối tích luỹ $(\mathrm{CDF})$ và hàm mật độ xác suất $(\mathrm{PDF})$ của các biến ngẫu nhiên $\gamma_{\mathrm{XY}}\left(\mathrm{X}, \mathrm{Y} \in\left\{\mathrm{S}, \mathrm{R}_{m}, \mathrm{D}, \mathrm{E}_{k}, \mathrm{P}_{n}\right\}\right)$ có thể lần lượt được đưa ra như trong biểu thức (1):

$$
\begin{aligned}
& F_{\gamma_{\mathrm{XY}}}(x)=1-\exp \left(-\lambda_{\mathrm{XY}} x\right), \\
& f_{\gamma_{\mathrm{XY}}}(x)=\lambda_{\mathrm{XY}} \exp \left(-\lambda_{\mathrm{XY}} x\right),
\end{aligned}
$$

trong đó $\lambda_{\mathrm{XY}}=1 / E\left\{\gamma_{\mathrm{XY}}\right\}$ với $E\left\{\gamma_{\mathrm{XY}}\right\}$ là giá trị trung bình của $\gamma_{X Y}$.

Để thuận tiện cho việc trình bày và phân tích, ta giả sử các biến ngẫu nhiên $\gamma_{\mathrm{SR}_{m}}, \gamma_{\mathrm{R}_{m} \mathrm{D}}, \gamma_{\mathrm{SP}_{n}}, \gamma_{\mathrm{R}_{m} \mathrm{P}_{n}}$ và $\gamma_{\mathrm{R}_{m} \mathrm{E}_{k}}$ là độc lập và đồng nhất, nghĩa là $\lambda_{\mathrm{SR}_{m}}=\lambda_{\mathrm{SR}}$, $\lambda_{\mathrm{R}_{m} \mathrm{D}}=\lambda_{\mathrm{RD}}, \lambda_{\mathrm{SP}_{n}}=\lambda_{\mathrm{SP}}, \lambda_{\mathrm{R}_{m} \mathrm{P}_{n}}=\lambda_{\mathrm{RP}}$ và $\lambda_{\mathrm{R}_{m} \mathrm{E}_{k}}=\lambda_{\mathrm{EK}}$, với mọi giá trị của $m, n$ và $k$. Hơn nữa, chúng tôi cũng xin lưu ý rằng, các phương pháp phân tích và đánh giá trong bài báo này hoàn toàn có thể được áp dụng trong các trường hợp mà các biến ngẫu nhiên không đồng nhất.

Giả sử rằng tất cả các nút trong hệ thống sơ cấp và thứ cấp đều được trang bị với một anten và hoạt động dưới chế độ bán song công (Half duplex). Do đó, hoạt động chuyển tiếp dữ liệu từ nguồn tới đích được thực hiện trên hai khe thời gian trực giao. Trong khe thời gian đầu, nút nguồn $\mathrm{S}$ sẽ phát quảng bá dữ liệu tới tất cả các nút chuyển tiếp. Tuy nhiên, trước khi truyền tin, nút nguồn phải điều chỉnh công suất phát để giao thoa tác động lên các nút sơ cấp không làm ảnh hưởng đến chất lượng dịch vụ của mạng sơ cấp. Tương tự như [18, công thức (8)], dưới sự xuất hiện của khiếm khuyết phần cứng và định mức giao thoa $I$, công suất phát tối đa mà nút nguồn có thể sử dụng được tính bởi:
$P_{\mathrm{S}}=\frac{I}{\left(1+\kappa_{\mathrm{P}}\right) \max _{n=1,2, \ldots, N}\left(\gamma_{\mathrm{SP}_{n}}\right)}=\frac{I}{\left(1+\kappa_{\mathrm{P}}\right) X_{\max }}$,

với $X_{\max }=\max _{n=1,2, \ldots, N}\left(\gamma_{\mathrm{SP}_{n}}\right)$ và $\kappa_{\mathrm{P}}$ là tổng mức suy hao phần cứng bao gồm cả sự suy hao tại nguồn và tại các nút sơ cấp.

Tiếp theo, tỷ số công suất tín hiệu trên nhiễu tức thời ( $\mathrm{SNR}$ ) của đường truyền từ $\mathrm{S} \rightarrow \mathrm{R}_{m}$ sẽ được biểu diễn dạng (3):

$$
\Psi_{\mathrm{SR}_{m}}=\frac{P_{\mathrm{S}_{\mathrm{SR}_{m}}}}{\kappa_{\mathrm{D}} P_{\mathrm{S}_{\mathrm{SR}_{m}}}+\sigma^{2}}=\frac{Q \gamma_{\mathrm{SR}_{m}} / X_{\max }}{\kappa_{\mathrm{D}} Q \gamma_{\mathrm{SR}_{m}} / X_{\text {max }}+1},
$$

ở đây $\sigma^{2}$ là phương sai của nhiễu cộng (nhiễu Gauss) tại nút chuyển tiếp $\mathrm{R}_{m}, Q=I /\left(1+\kappa_{\mathrm{P}}\right) / \sigma^{2}$ và $\kappa_{\mathrm{D}}$ là tổng mức suy hao phần cứng trên kênh dữ liệu từ $\mathrm{S}$ đến $\mathbf{R}_{m}$.

Nút $\mathbf{R}_{m}$ được giả sử là nhận thành công dữ liệu từ nguồn nếu nút tỷ số công suất tín hiệu trên nhiễu $\Psi_{\mathrm{SR}_{m}}$ cao hơn mức ngưỡng $\gamma_{t h}$. Ngược lại, nút này được xem là không thể giải mã dữ liệu thành công và xem như rơi vào trạng thái dừng.

Sau khi nhận được tín hiệu từ nguồn, tất cả các nút chuyển tiếp cố gắng giải mã dữ liệu. Không mất tính tổng quát, ta có thể giả sử rằng $\mathrm{R}_{1}, \mathrm{R}_{2}, \ldots, \mathrm{R}_{t}$ là các nút chuyển tiếp giải mã thành công, trong khi $\mathrm{R}_{t+1}, \mathrm{R}_{t+2}, \ldots, \mathrm{R}_{M}$ là các nút nhận không thể giải mã được, với $t$ là một số nguyên chạy từ 0 đến $M$. Khi $t=$ 0 có nghĩa là không có nút chuyển tiếp nào giải mã được dữ liệu nguồn, và trong trường hợp này hệ thống sẽ bị dừng vì nút đích không thể nhận được dữ liệu từ nguồn. Ta có thể nhận thấy rằng, $t$ là một biến ngẫu nhiên và xác suất mà số nút chuyển tiếp thành công bằng $t$ được tính bằng biểu thức (4) như sau:

$$
A_{t}=C_{M}^{t} \operatorname{Pr}\left(\begin{array}{c}
\Psi_{\mathrm{SR}_{1}} \geq \gamma_{t h}, \ldots, \Psi_{\mathrm{SR}_{t}} \geq \gamma_{t h}, \\
\Psi_{\mathrm{SR}_{t+1}}<\gamma_{t h}, \ldots, \Psi_{\mathrm{SR}_{M}}<\gamma_{t h}
\end{array}\right) .
$$

Thay (3) vào (4), ta được công thức (5) như sau: 


$$
A_{t}=C_{M}^{t} \operatorname{Pr}\left(\begin{array}{c}
\gamma_{\mathrm{SR}_{1}} \geq \chi_{\mathrm{D}} X_{\text {max }}, \ldots, \gamma_{\mathrm{SR}_{t}} \geq \chi_{\mathrm{D}} X_{\text {max }}, \\
\gamma_{\mathrm{SR}_{t+1}}<\chi_{\mathrm{D}} X_{\text {max }}, \ldots, \gamma_{\mathrm{SR}_{M}}<\chi_{\mathrm{D}} X_{\text {max }}
\end{array}\right),
$$

với $\chi_{\mathrm{D}}=\gamma_{t h} / Q /\left(1-\kappa_{\mathrm{D}} \gamma_{t h}\right)$. Ở đây, ta giả sử rằng mức suy hao phần cứng $\kappa_{\mathrm{D}}$ đủ nhỏ để mà $\kappa_{\mathrm{D}}<1 / \gamma_{t h}$. Ta không xét trường hợp $\kappa_{\mathrm{D}} \geq 1 / \gamma_{\text {th }}$ bởi vì hệ thống khảo sát luôn bị dừng trong trường hợp này [14], [15].

Xét sự truyền dữ liệu ở khe thời gian thứ hai, một trong những nút chuyển tiếp thành công $\left(\mathrm{R}_{1}, \mathrm{R}_{2}, \ldots, \mathrm{R}_{t}\right)$. Bây giờ, chúng tôi sẽ lần lượt giới thiệu các phương pháp lựa chọn nút chuyển tiếp.

Trong giao thức đề xuất đầu tiên với tên gọi HCG$\mathrm{D}$, nút chuyển tiếp được chọn là nút có độ lợi kênh truyền đến nút đích lớn nhất. Thật vậy, sự chọn lựa được thực hiện theo thuật toán sau:

$$
\mathrm{R}_{b}: \gamma_{\mathrm{R}_{b} \mathrm{D}}=\max _{v=1,2, \ldots, t} \gamma_{\mathrm{R}_{v} \mathrm{D}},
$$

trong đó $\mathrm{R}_{b}$ là nút chuyển tiếp được chọn.

Tương tự (2), trước khi phát dữ liệu tới nút đích, nút $\mathrm{R}_{b}$ điều chỉnh công suất phát như công thức (7):

$$
P_{\mathrm{R}_{b}}=\frac{I}{\left(1+\kappa_{\mathrm{P}}\right) \max _{n=1,2, \ldots, N}\left(\gamma_{\mathrm{R}_{b} \mathrm{P}_{n}}\right)}=\frac{I}{\left(1+\kappa_{\mathrm{P}}\right) Y_{b, \max }},
$$

với $Y_{b, \max }=\max _{n=1,2, \ldots, N}\left(\gamma_{\mathrm{R}_{b} \mathrm{P}_{n}}\right)$.

Rồi thì, tỷ số công suất tín hiệu trên nhiễu tức thời nhận được tại nút đích được tính bởi công thức (8):

$$
\Psi_{\mathrm{R}_{b} \mathrm{D}}=\frac{Q \gamma_{\mathrm{R}_{b} \mathrm{D}} / Y_{b, \max }}{\kappa_{\mathrm{D}} Q \gamma_{\mathrm{R}_{b} \mathrm{D}} / Y_{b, \text { max }}+1} .
$$

Ta có nhận xét rằng tỷ số tín hiệu trên nhiễu lớn nhất nhận được tại các nút nghe lén sẽ đặc trưng cho tỷ số tín hiệu trên nhiễu của kênh nghe lén (xem [16, công thức (8)]). Kí hiệu $\Psi_{\mathrm{R}_{b} \mathrm{E}}$ là tỷ số tín hiệu trên nhiễu của kênh nghe lén, ta có:

$$
\begin{aligned}
\Psi_{\mathrm{R}_{b} \mathrm{E}} & =\max _{k=1,2, \ldots, K}\left(\frac{Q \gamma_{\mathrm{R}_{b} \mathrm{E}_{k}} / Y_{b, \text { max }}}{\kappa_{\mathrm{E}} Q \gamma_{\mathrm{R}_{b} \mathrm{E}_{k}} / Y_{b, \text { max }}+1}\right) \\
& =\frac{Q Z_{b, \text { max }} / Y_{b, \text { max }}}{\kappa_{\mathrm{E}} Q Z_{b, \text { max }} / Y_{b, \text { max }}+1},
\end{aligned}
$$

với $\kappa_{\mathrm{E}}$ là tổng mức suy hao phần cứng trên kênh nghe lén và $Z_{b, \max }=\max _{k=1,2, \ldots, K}\left(\gamma_{\mathrm{R}_{b} \mathrm{E}_{k}}\right)$.

Từ các công thức (8) và (9), xác suất dừng tại nút đích $\mathrm{D}(\mathrm{OP})$ và khả năng giải mã tại các nút nghe lén $\mathrm{E}$ (DP) có thể được đưa ra như biểu thức (10):

$$
\begin{aligned}
& \mathrm{OP}_{\mathrm{HCG}-\mathrm{D}}=\operatorname{Pr}\left(\Psi_{\mathrm{R}_{b} \mathrm{D}}<\gamma_{t h}\right), \\
& \mathrm{DP}_{\text {HCG-D }}=\operatorname{Pr}\left(\Psi_{\mathrm{R}_{b} \mathrm{E}} \geq \gamma_{t h}\right) .
\end{aligned}
$$

Mặc dù giao thức HCG-D nâng cao chất lượng đường truyền dữ liệu giữa nút chuyển tiếp và nút đích, nhưng kỹ thuật này không giảm khả năng giải mã của các kênh nghe lén. Chúng ta có thể thấy rằng, để giảm chất lượng kênh truyền nghe lén, hệ thống phải chọn nút chuyển tiếp đạt được tỷ số tín hiệu trên nhiễu đối với kênh nghe lén là thấp nhất. Do đó, nút chuyển tiếp được chọn trong giao thức MCG-E được đưa ra bởi công thức (11):

$$
\mathrm{R}_{c}: \max _{k=1,2, . ., K}\left(\gamma_{\mathrm{R}_{c} \mathrm{E}_{k}}\right)=\min _{v=1,2, \ldots, t}\left[\max _{k=1,2, . ., K}\left(\gamma_{\mathrm{R}_{v} \mathrm{E}_{k}}\right)\right] .
$$

với $\mathrm{R}_{c}$ là nút chuyển tiếp được chọn.

Tương tự, tỷ số tín hiệu trên nhiễu tức thời nhận được tại nút đích và các nút nghe lén trong giao thức MCG-E có thể lần lượt được diễn đạt như trong biểu thức (12) bên dưới:

$$
\begin{aligned}
& \Psi_{\mathrm{R}_{c} \mathrm{D}}=\frac{Q \gamma_{\mathrm{R}_{c} \mathrm{D}} / Y_{c, \text { max }}}{\kappa_{\mathrm{D}} Q \gamma_{\mathrm{R}_{c} \mathrm{D}} / Y_{c, \text { max }}+1}, \\
& \Psi_{\mathrm{R}_{c} \mathrm{E}}=\frac{Q Z_{c, \text { max }} / Y_{c, \text { max }}}{\kappa_{\mathrm{E}} Q Z_{c, \text { max }} / Y_{c, \text { max }}+1},
\end{aligned}
$$

với $Z_{c, \text { max }}=\max _{k=1,2, \ldots, K}\left(\gamma_{\mathrm{R}_{c} \mathrm{E}_{k}}\right)$ và $Y_{c, \text { max }}=\max _{n=1,2, \ldots, N}\left(\gamma_{\mathrm{R}_{c} \mathrm{P}_{n}}\right)$.

Từ (12), các giá trị $\mathrm{OP}$ và $\mathrm{DP}$ của giao thức MCGE được tính bởi biểu thức (13): 


$$
\begin{aligned}
& \mathrm{OP}_{\text {MCG-E }}=\operatorname{Pr}\left(\Psi_{\mathrm{R}_{c} \mathrm{D}}<\gamma_{t h}\right), \\
& \mathrm{DP}_{\text {MCG-E }}=\operatorname{Pr}\left(\Psi_{\mathrm{R}_{c} \mathrm{E}} \geq \gamma_{t h}\right) .
\end{aligned}
$$

Tuy nhiên, giao thức MCG-E chỉ tập trung làm giảm chất lượng kênh nghe lén mà không quan tâm đến việc nâng cao chất lượng kênh dữ liệu. Trong giao thức $\mathrm{COMB}$, phương pháp chọn lựa nút chuyển tiếp sẽ được trình bày cụ thể như sau:

Xét tập các nút chuyển tiếp nhận dữ liệu nguồn thành công: $\mathrm{R}_{1}, \mathrm{R}_{2}, \ldots, \mathrm{R}_{t}$, không mất tính tổng quát, ta giả sử rằng: $\gamma_{\mathrm{R}_{1} \mathrm{D}} \geq \gamma_{\mathrm{R}_{2} \mathrm{D}} \geq \ldots \geq \gamma_{\mathrm{R}_{t} \mathrm{D}}$. Đầu tiên, hệ thống sẽ chọn ra một nhóm các nút chuyển tiếp này có độ lợi kênh truyền cao nhất như sau: $\mathrm{R}_{1}, \mathrm{R}_{2}, \ldots, \mathrm{R}_{\mathrm{L}}$, trong đó, $L$ được xác định bởi: $L=\lceil t / 2\rceil$ với $\lceil t / 2\rceil$ là số nguyên nhỏ nhất lớn hơn hoặc bằng $t / 2$. Từ tập $L$ nút chuyển tiếp này, giao thức COMB sẽ chọn ra một nút chuyển tiếp theo thuật toán được diễn đạt như trong (14):

$$
\mathrm{R}_{d}: \max _{k=1,2, \ldots, K}\left(\gamma_{\mathrm{R}_{d} \mathrm{E}_{k}}\right)=\min _{u=1,2, \ldots, L}\left[\max _{k=1,2, \ldots, K}\left(\gamma_{\mathrm{R}_{u} \mathrm{E}_{k}}\right)\right] .
$$

Ta thấy rằng, giao thức $\mathrm{COMB}$ sẽ nâng cao chất lượng kênh dữ liệu của việc chọn nút chuyển tiếp từ tập các nút có độ lợi kênh đến nút đích lớn. Hơn thế nữa, bằng việc chọn nút chuyển tiếp có độ lợi kênh thấp đến các nút $\mathrm{E}, \mathrm{COMB}$ cũng làm giảm chất lượng kênh nghe lén.

Tương tự, các tỷ số công suất tín hiệu trên nhiễu tức thời (SNR) tại nút nghe lén và nút đích trong mô hình COMB lần lượt đạt được bởi (15):

$$
\begin{aligned}
& \Psi_{\mathrm{R}_{d} \mathrm{D}}=\frac{Q \gamma_{\mathrm{R}_{d} \mathrm{D}} / Y_{d, \text { max }}}{\kappa_{\mathrm{D}} Q \gamma_{\mathrm{R}_{d} \mathrm{D}} / Y_{d, \text { max }}+1}, \\
& \Psi_{\mathrm{R}_{d} \mathrm{E}}=\frac{Q Z_{d, \text { max }} / Y_{d, \text { max }}}{\kappa_{\mathrm{E}} Q Z_{d, \text { max }} / Y_{d, \text { max }}+1} .
\end{aligned}
$$

Từ đây, các xác suất $\mathrm{OP}$ và $\mathrm{DP}$ được viết dưới công thức (16):

$$
\begin{aligned}
& \mathrm{OP}_{\mathrm{COMB}}=\operatorname{Pr}\left(\Psi_{\mathrm{R}_{d} \mathrm{D}}<\gamma_{t h}\right), \\
& \mathrm{DP}_{\mathrm{COMB}}=\operatorname{Pr}\left(\Psi_{\mathrm{R}_{d} \mathrm{E}}<\gamma_{t h}\right) .
\end{aligned}
$$

Cuối cùng, chúng tôi đưa ra công thức tổng quát tính giá trị trung bình của $\mathrm{OP}$ và $\mathrm{DP}$ cho giao thức $\mathrm{X}(\mathrm{X} \in\{\mathrm{HCG}-\mathrm{D}, \mathrm{MCG}-\mathrm{E}, \mathrm{COMB}\})$ được đưa ra bằng một công thức hợp nhất như trong (17):

$$
\begin{aligned}
& \overline{\mathrm{OP}}_{\mathrm{X}}=\sum_{t=0}^{M} A_{t} \mathrm{OP}_{\mathrm{X}}, \\
& \overline{\mathrm{DP}}_{\mathrm{X}}=\sum_{t=1}^{M} A_{t} \mathrm{DP}_{\mathrm{X}} .
\end{aligned}
$$

\section{III. ĐÁNH GIÁ HIỆU NĂNG HỆ THỐNG}

\section{III.1. Chuẩn bị toán học}

Đầu tiên, ta xét biến ngẫu nhiên $U$ với $U=$ $\max \left(\gamma_{1}, \gamma_{2}, \ldots, \gamma_{V}\right)$ và $V$ là một số tự nhiên lớn hơn hoặc bằng $1 . \gamma_{1}, \gamma_{2}, \ldots, \gamma_{V-1}$ và $\gamma_{V}$ là các biến ngẫu nhiên có phân bố mũ và có cùng tham số đặc trưng $\lambda=1 / E\left\{\gamma_{v}\right\}, \forall v \in\{1,2, \ldots, V\}$. Sử dụng [19, công thức (12)], ta viết ra hàm PDF của $U$ như:

$$
f_{U}(x)=\sum_{v=0}^{V-1}(-1)^{v} C_{V}^{v} V \lambda \exp [-(v+1) \lambda x] .
$$

Hơn nữa, với [19, công thức (11)], ta có thể đưa ra hàm $\mathrm{CDF}$ của $U$.

$$
F_{U}(x)=1+\sum_{v=1}^{V}(-1)^{v} C_{V}^{v} \exp (-v \lambda x) .
$$

Tiếp theo, ta nghiên cứu các hàm phân bố của biến ngẫu nhiên $W, W=\min _{i=1,2, \ldots, R} \max _{j=1,2, \ldots, V}\left(\gamma_{i j}\right)$, ở đây $\gamma_{i j}$ cũng là các biến ngẫu nhiên có phân phối mũ và có cùng tham số đặc trưng $\lambda$. Cũng vậy, $R$ là một số tự nhiên lớn hơn hoặc bằng 1 . Tiếp theo, ta có thể xây dựng hàm CDF cho biến ngẫu nhiên $W$ bởi: 


$$
\begin{aligned}
F_{W}(x) & =\operatorname{Pr}(W<x) \\
& =1-\left\{1-[1-\exp (-\lambda x)]^{V}\right\}^{R} \\
& =\sum_{r=1}^{R}(-1)^{r+1} C_{R}^{r}[1-\exp (-\lambda x)]^{r V} \\
& =\sum_{r=1}^{R} \sum_{v=0}^{r V}(-1)^{r+v+1} C_{R}^{r} C_{r V}^{v} \exp (-v \lambda x) .
\end{aligned}
$$

Xét độ lợi kênh truyền $\gamma_{\mathrm{R}_{d} \mathrm{D}}$ trong giao thức $\mathrm{COMB}$, chúng ta thấy rằng $\gamma_{\mathrm{R}_{d} \mathrm{D}}$ nhận giá trị lớn nhất trong tập các biến ngẫu nhiên $\left\{\gamma_{\mathrm{R}_{1} \mathrm{D}}, \gamma_{\mathrm{R}_{2} \mathrm{D}}, \ldots, \gamma_{\mathrm{R}_{t} \mathrm{D}}\right\}$ khi $d=1$. Khi $d=2$ thì $\gamma_{\mathrm{R}_{d} \mathrm{D}}$ đạt giá trị lớn thứ hai, ... và $\gamma_{\mathrm{R}_{d} \mathrm{D}}$ có giá trị cao thứ $\mathrm{L}$ khi $d=L$. khi $d=l$, với $l=$ $1,2, \ldots, L$, sử dụng thống kê bậc thứ $l[20$, phương trình (5)], hàm phân phối tích lũy của $\gamma_{\mathrm{R}_{d} \mathrm{D}}$ có thể được viết ra trong công thức (21):

$$
\begin{aligned}
F_{\gamma_{\mathrm{R}_{d} \mathrm{D}}}(x)= & \sum_{u=1}^{l} \sum_{v=0}^{t-u+1}(-1)^{v} C_{t}^{u-1} C_{t-u+1}^{v} \\
& \times \exp \left[-(u+v-1) \lambda_{\mathrm{RD}} x\right] .
\end{aligned}
$$

Hơn nữa, bởi sự đồng nhất của các biến ngẫu nhiên, xác suất $\gamma_{\mathrm{R}_{d} \mathrm{D}}$ nhận giá trị lớn thứ $l$ sẽ là:

$$
\operatorname{Pr}(d=l)=\frac{1}{L} \text {. }
$$

\section{III.2. Tính giá trị $A_{t}$ trong công thức (5)}

Từ (5), ta có thể viết lại $A_{t}$ dưới dạng công thức (23) bên dưới:

$$
\begin{aligned}
A_{t}= & C_{M}^{t} \int_{0}^{\infty}\left[1-F_{\gamma_{\mathrm{SR}_{m}}}\left(\chi_{\mathrm{D}} x\right)\right]^{t} \\
& \times\left[F_{\gamma_{\mathrm{SR}_{m}}}\left(\chi_{\mathrm{D}} x\right)\right]^{M-t} f_{X_{\max }}(x) d x .
\end{aligned}
$$

Thay hàm $\mathrm{CDF}$ của $\gamma_{\mathrm{SR}_{m}}$ trong công thức (1) và hàm $\mathrm{PDF}$ của $X_{\max }$ trong công thức (18) vào công thức (23) ở trên; sau vài bước biến đổi và tính toán, ta đạt được rằng:

$$
\begin{aligned}
A_{t}= & C_{M}^{t} \sum_{u=0}^{M-t} \sum_{v=0}^{N-1}(-1)^{u+v} C_{M-t}^{u} C_{N-1}^{v} \\
& \times \frac{N \lambda_{\mathrm{SP}}}{(v+1) \lambda_{\mathrm{SP}}+(u+t) \lambda_{\mathrm{SR}} \chi_{\mathrm{D}}} .
\end{aligned}
$$

\section{III.3. Tính giá trị $\mathrm{OP}$ và $\mathrm{DP}$ của các giao thức}

Xét phương pháp đề xuất đầu tiên HCG-D, xác suất dừng $\mathrm{OP}_{\mathrm{HCG}-\mathrm{D}}$ trong $(10)$ có thể được đưa ra dưới dạng biểu thức (25):

$$
\begin{aligned}
\mathrm{OP}_{\mathrm{HCG}-\mathrm{D}} & =\operatorname{Pr}\left(\gamma_{\mathrm{R}_{b} \mathrm{D}}<\chi_{\mathrm{D}} Y_{b, \text { max }}\right) \\
& =\int_{0}^{+\infty} F_{\gamma_{\mathrm{R}_{b} \mathrm{D}}}\left(\chi_{\mathrm{D}} x\right) f_{Y_{b, \text { max }}}(x) d x .
\end{aligned}
$$

Thay hàm CDF của $F_{\gamma_{\mathrm{R} b \mathrm{D}}}\left(\chi_{\mathrm{D}} x\right)$ như trong (19) và hàm $\mathrm{PDF}$ của $f_{Y_{b, \text { max }}}(x)$ như trong (18) vào công thức (25) sau vài bước tính toán, ta tính được chính xác $\mathrm{OP}_{\mathrm{HCG}-\mathrm{D}}$ như trong công thức (26):

$$
\begin{aligned}
\mathrm{OP}_{\mathrm{HCG}-\mathrm{D}}=1 & +\sum_{v=1}^{t} \sum_{n=0}^{N-1}(-1)^{v+n} C_{t}^{v} C_{N-1}^{n} \\
& \times \frac{N \lambda_{\mathrm{RP}}}{(n+1) \lambda_{\mathrm{RP}}+v \lambda_{\mathrm{RD}} \chi_{\mathrm{D}}} .
\end{aligned}
$$

Một cách tương tự, khả năng giải mã của các nút nghe lén thứ cấp trong giao thức HCG-D cũng được đưa về dạng biểu thức (27):

$$
\begin{aligned}
\mathrm{DP}_{\mathrm{HCG}-\mathrm{D}} & =\operatorname{Pr}\left(\gamma_{\mathrm{R}_{b} \mathrm{E}} \geq \chi_{\mathrm{E}} Y_{b, \text { max }}\right) \\
& =\int_{0}^{+\infty}\left[1-F_{\gamma_{\mathrm{R}_{b} \mathrm{E}}}\left(\chi_{\mathrm{E}} x\right)\right] f_{Y_{b, \text { max }}}(x) d x,
\end{aligned}
$$

với $\chi_{\mathrm{E}}=\gamma_{t h} / Q /\left(1-\kappa_{\mathrm{E}} \gamma_{t h}\right)$. Cũng vậy, trong bài báo, ta cũng chỉ xét trường hợp $\kappa_{\mathrm{E}}<1 / \gamma_{t h}$, bởi vì nếu $\kappa_{\mathrm{E}} \geq 1 / \gamma_{t h}$, các nút nghe lén sẽ không thể giải mã được dữ liệu với mọi giá trị của các tham số khác.

Một lần nữa, ta sử dụng hàm $\mathrm{CDF}$ trong (19) cho $F_{\gamma_{\mathrm{R}_{b} \mathrm{E}}}\left(\chi_{\mathrm{E}} x\right)$ và hàm PDF trong (18) cho $f_{Y_{b, \max }}(x)$, để tính chính xác $\mathrm{P}_{\text {HCG-D }}$ như trong công thức (28) sau:

$$
\begin{aligned}
\mathrm{DP}_{\mathrm{HCG}-\mathrm{D}}= & \sum_{k=1}^{K} \sum_{n=0}^{N-1}(-1)^{k+n+1} C_{K}^{k} C_{N-1}^{n} \\
& \times \frac{N \lambda_{\mathrm{RP}}}{(n+1) \lambda_{\mathrm{RP}}+k \lambda_{\mathrm{RE}} \chi_{\mathrm{E}}} .
\end{aligned}
$$


Bây giờ, thay các kết quả thu được từ (23), (26) và (28) vào trong (17), ta thu được các biểu thức dạng đóng chính xác cho các giá trị trung bình của $\mathrm{OP}$ và IP của giao thức HCG-D.

Tiếp đến, xét giao thức MCG-E, xác suất dừng của giao thức này có thể đạt được như trong (29):

$$
\begin{aligned}
\mathrm{OP}_{\mathrm{MCG}-\mathrm{E}} & =\operatorname{Pr}\left(\gamma_{\mathrm{R}_{c} \mathrm{D}}<\chi_{\mathrm{D}} Y_{c, \text { max }}\right) \\
& =1-\sum_{n=0}^{N-1}(-1)^{n} \frac{C_{N-1}^{n} N \lambda_{\mathrm{RP}}}{(n+1) \lambda_{\mathrm{RP}}+\lambda_{\mathrm{RD}} \chi_{\mathrm{D}}} .
\end{aligned}
$$

Xét giá trị $\mathrm{DP}_{\mathrm{MCG}-\mathrm{E}}$, sử dụng hàm $\mathrm{CDF}$ đạt được trong (20) cho $F_{\gamma_{\mathrm{R}_{\mathrm{E}}}}(x)$, ta có:

$$
\begin{aligned}
\mathrm{DP}_{\mathrm{MCG}-\mathrm{E}}= & \operatorname{Pr}\left(\gamma_{\mathrm{R}_{c} \mathrm{E}} \geq \chi_{\mathrm{E}} Y_{c, \text { max }}\right) \\
= & \int_{0}^{+\infty}\left[1-F_{\gamma_{\mathrm{R}_{c} \mathrm{E}}}\left(\chi_{\mathrm{E}} x\right)\right] f_{Y_{c, \text { max }}}(x) d x n \\
= & 1-\sum_{v=1}^{t} \sum_{u=0}^{v K} \sum_{n=0}^{N-1}(-1)^{v+u+n+1} C_{t}^{v} C_{v k}^{u} C_{N-1}^{n} \\
& \times \frac{N \lambda_{\mathrm{RP}}}{(n+1) \lambda_{\mathrm{RP}}+u \lambda_{\mathrm{RE}}} .
\end{aligned}
$$

Từ các kết quả đạt được trong (23), (29) và (30), ta dễ dàng tính được $\overline{\mathrm{OP}}_{\text {MCG-E }}$ và $\overline{\mathrm{DP}}_{\text {MCG-E }}$.

Đối với giao thức cuối cùng $(\mathrm{COMB})$, biểu thức của $\mathrm{OP}_{\mathrm{COMB}}$ có thể được viết lại như trong công thức (31):

$$
\begin{aligned}
& \mathrm{OP}_{\mathrm{COMB}}=\operatorname{Pr}\left(\gamma_{\mathrm{R}_{d} \mathrm{D}} \leq \chi_{\mathrm{D}} Y_{d, \text { max }}\right) \\
& =\sum_{l=1}^{L} \operatorname{Pr}(d=l) \int_{0}^{+\infty} F_{\gamma_{\mathrm{R}_{d} \mathrm{D}}}\left(\chi_{\mathrm{D}} x\right) f_{Y_{d, \text { max }}}(x) d x .
\end{aligned}
$$

Sử dụng hàm CDF đạt được trong công thức (21) và hàm $P D F$ được đưa ra trong công thức (18), sau vài bước biến đổi và tính toán, ta đạt được kết quả bởi biểu thức (32):

$$
\begin{aligned}
\mathrm{OP}_{\mathrm{COMB}}= & \frac{1}{L} \sum_{l=1}^{L} \sum_{u=1}^{l} \sum_{v=0}^{t-u+1} \sum_{n=0}^{N-1}(-1)^{v+n} \\
& \times \frac{C_{t}^{u-1} C_{t-u+1}^{v} C_{N-1}^{n} N \lambda_{\mathrm{RP}}}{(n+1) \lambda_{\mathrm{RP}}+(u+v-1) \lambda_{\mathrm{RD}} \chi_{\mathrm{D}}} .
\end{aligned}
$$

Trong giao thức $\mathrm{COMB}$ này, khả năng giải mã của các nút nghe lén được tính tương tự như (30) và được biểu diễn bởi công thức (33):

$$
\begin{aligned}
\mathrm{DP}_{\mathrm{COMB}}= & \operatorname{Pr}\left(\gamma_{\mathrm{R}_{d} \mathrm{E}} \geq \chi_{\mathrm{E}} Y_{d, \max }\right) \\
=1+ & \sum_{v=1}^{L} \sum_{u=0}^{v K} \sum_{n=0}^{N-1}(-1)^{v+u+n+1} C_{L}^{v} C_{v K}^{u} C_{N-1}^{n} \\
& \times \frac{N \lambda_{\mathrm{RP}}}{(n+1) \lambda_{\mathrm{RP}}+u \lambda_{\mathrm{RE}}} .
\end{aligned}
$$

Cuối cùng, với các kết quả đạt được trong các công thức (23), (32) và (33), ta dễ dàng tính được xác suất dừng trung bình $\overline{\mathrm{OP}}_{\text {Сомв và }} \overline{\mathrm{DP}}_{\mathrm{COMB}}$ như được đưa ra trong (17).

\section{KẾT QUẢ MÔ PHỎNG}

Trong phần này, chúng tôi thực hiện mô phỏng Monte Carlo để kiểm chứng các kết quả lý thuyết được đưa ra trong Phần trước. Để các kết quả mô phỏng hội tụ về các kết quả lý thuyết, chúng tôi đã thực hiện $10^{6}$ phép thử cho mỗi kết quả mô phỏng. Hơn nữa, chúng tôi cố định tất cả các tham số đặc trưng bằng $1: \lambda_{\mathrm{SR}}=\lambda_{\mathrm{RD}}=\lambda_{\mathrm{SP}}=\lambda_{\mathrm{RD}}=\lambda_{\mathrm{RP}}=1$, và giá trị của ngưỡng dừng $\gamma_{t h}$ cũng không thay đổi $\left(\gamma_{t h}=1\right)$ trong các mô phỏng.

Hình 2 biểu diễn giá trị xác suất dừng $(\mathrm{OP})$ như một hàm số của $Q$. Trong mô phỏng này, số lượng nút chuyển tiếp $(M)$ bằng 3 và 6 , số nút nghe lén $(K)$ bằng 2 và số các nút thứ cấp $N$ bằng 3 . Các thông số suy hao phần cứng được thiết lập như sau: $\kappa_{\mathrm{P}}=0.1$ và $\kappa_{\mathrm{P}}=\kappa_{\mathrm{P}}=0.5$. Kết quả trong Hình 2 cho thấy rằng xác suất dừng $(\mathrm{OP})$ của tất cả các giao thức giảm khi giá trị $Q$ tăng. Mặc khác, giá trị OP trong giao thức HCG$\mathrm{D}$ là thấp nhất bởi giao thức này chọn nút chuyển tiếp có độ lợi kênh truyền lớn nhất tới đích. Giá trị $\mathrm{OP}$ của mô hình MCG-E là lớn nhất bởi mô hình này chỉ quan tâm đến việc tối thiểu hóa chất lượng kênh nghe lén. Mô hình $\mathrm{COMB}$ đạt được hiệu năng nằm giữa hai mô hình HCG-D và MCG-E. Cũng quan sát trên hình vẽ này, ta thấy rằng giá trị xác suất dừng của hai mô hình HCG-D và $C O M B$ giảm nhanh khi tăng số lượng nút chuyển tiếp từ 3 lên 6 . Tuy nhiên, đối với giao thức MCG-E, giá trị này chỉ giảm nhẹ khi thay đổi số nút chuyển tiếp. Một kết quả đáng chú ý nữa mà ta có thể 
thấy trên hình vẽ là các kết quả mô phỏng (MP) trùng khớp với các kết quả lý thuyết (LT), điều này chứng tỏ các công thức đưa ra trong Phần III là chính xác.

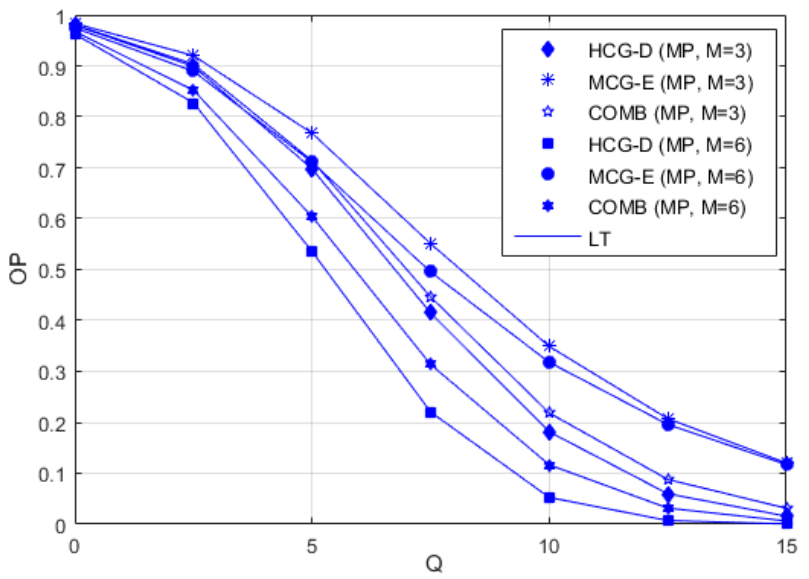

Hình 2. Xác suất dùng $(O P)$ theo $Q(d B)$ khi $N=3$, $K=2, \kappa_{\mathrm{P}}=\kappa_{\mathrm{E}}=0.5$ và $\kappa_{\mathrm{P}}=0.1$

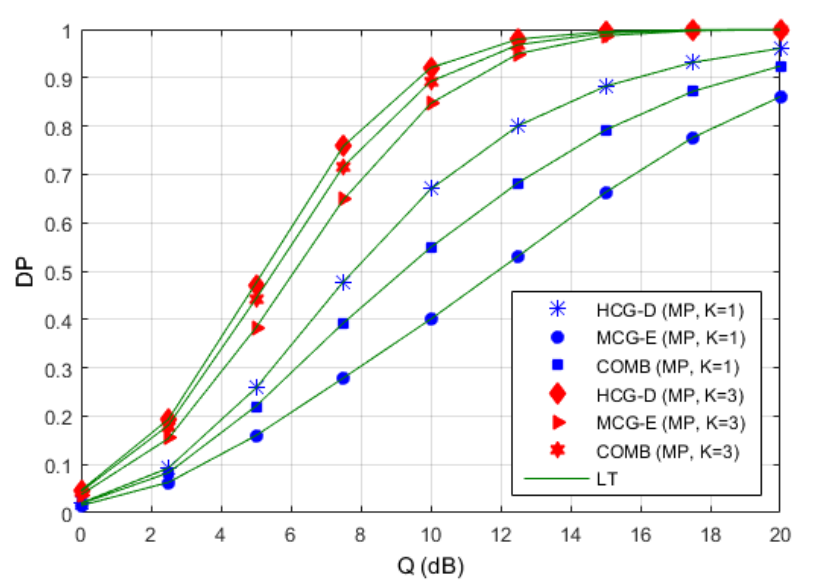

Hình 3. Khả năng giải mã của các nút nghe lén (DP) theo $Q(d B)$ khi $M=4, N=3, \kappa_{\mathrm{D}}=\kappa_{\mathrm{E}}=0.5$ và

$$
\kappa_{\mathrm{P}}=0.1
$$

Trong Hình 3, chúng tôi biểu diễn khả năng giải mã (DP) của các nút nghe lén theo giá trị của $Q$. Các thông số của hình vẽ này là $M=4, N=3$, $\kappa_{\mathrm{D}}=\kappa_{\mathrm{E}}=0.5$ và $\kappa_{\mathrm{P}}=0.1$. Quan sát hình vẽ, ta thấy rằng giá trị $\mathrm{DP}$ của giao thức $\mathrm{MCG}-\mathrm{E}$ là thấp nhất, trong khi giá trị $\mathrm{DP}$ trong giao thức $\mathrm{COMB}$ nằm giữa hai giao thức còn lại. Đó là vì các mô hình $\mathrm{MCG-E}$ và COMB quan tâm đến việc hạn chế chất lượng kênh nghe lén. Nhìn vào hình vẽ, ta cũng thấy rằng giá trị $\mathrm{DP}$ của tất cả các giao thức tăng với sự gia tăng của $Q$.
Hơn thế nữa, giá trị này tăng mạnh khi số lượng nút nghe lén tăng từ 1 lên 3 . Một lần nữa, các kết quả mô phỏng đã kiểm chứng sự chính xác của các biểu thức toán học đánh giá giá trị DP.

Hình 4 khảo sát sự biến thiên của giá trị xác suất dừng $(\mathrm{OP})$ theo số lượng nút sơ cấp $(N)$ khi $Q=5 \mathrm{~dB}$, $M=7, K=2, \kappa_{\mathrm{D}}=\kappa_{\mathrm{E}}=0.2$ và $\kappa_{\mathrm{P}}=0$. Nhìn vào hình vẽ, ta thấy rằng giá trị $\mathrm{OP}$ của các giao thức tăng theo sự gia tăng của số lượng nút sơ cấp. Đó là vì khi số lượng $N$ tăng sẽ làm giảm công suất phát của nút nguồn thứ cấp và các nút chuyển tiếp thứ cấp, kéo theo sự tăng của giá trị OP.

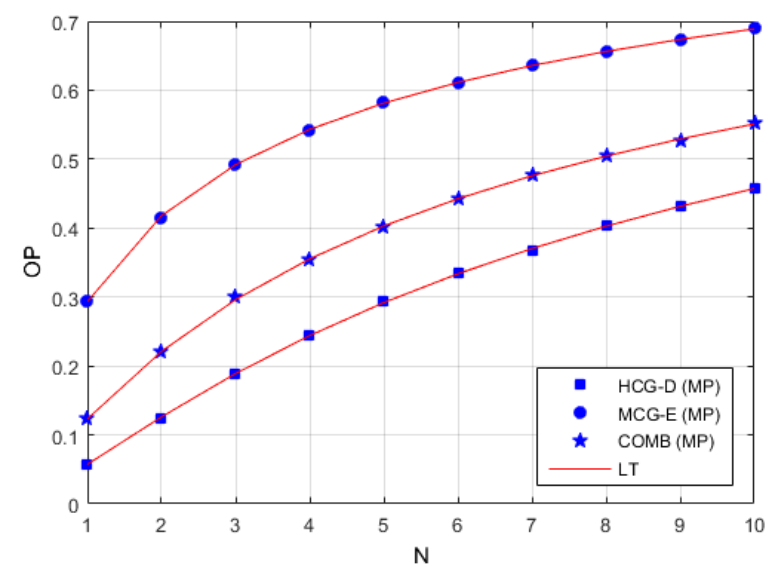

Hình 4. Xác suất dì̀ng (OP) theo N khi $Q=5 d B$, $M=7, K=2, \kappa_{\mathrm{D}}=\kappa_{\mathrm{E}}=0.2$ và $\kappa_{\mathrm{P}}=0$

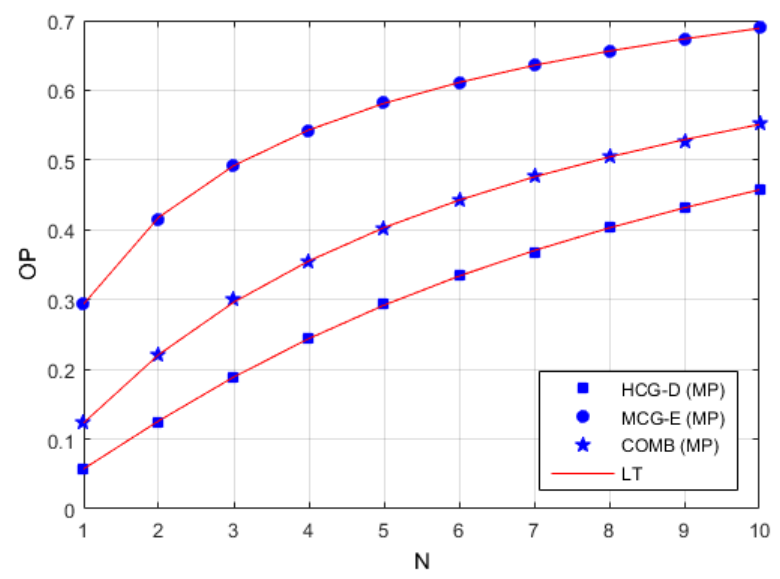

Hình 5. Xác suất dìng $(O P)$ theo $\kappa_{\mathrm{E}}$ khi $Q=5 \mathrm{~dB}$, $M=5, K=1, N=3, \kappa_{\mathrm{D}}=0.5$ và $\kappa_{\mathrm{P}}=0.1$ 
Trong Hình 5 , giá trị DP của các mô hình đề xuất được vẽ theo giá trị mức suy hao phần cứng $\kappa_{\mathrm{E}}$ khi $Q=5 \mathrm{~dB}, M=5, K=1, N=3, \kappa_{\mathrm{D}}=0.5$ và $\kappa_{\mathrm{P}}=0.1$. Hình 5 cho thấy rằng giá trị DP giảm mạnh khi tăng $\kappa_{\mathrm{E}}$ từ 0 đến 0.9 . Một lần nữa, ta có thể thấy từ Hình 4 và Hình 5 rằng mô hình $\mathrm{HCG-D}$ đạt hiệu năng $\mathrm{OP}$ tốt nhất, mô hình MCG-E nhận giá trị $\mathrm{DP}$ nhỏ nhất và hiệu năng của mô hình $\mathrm{COMB}$ nằm giữa hai mô hình còn lại.

Bảng 1. Giá trị của $Q(d B)$ trong Hình 6

\begin{tabular}{|c|c|c|c|c|c|c|}
\hline OP & $10^{-0.5}$ & $10^{-1}$ & $10^{-1.5}$ & $10^{-2}$ & $10^{-2.5}$ & $10^{-3}$ \\
\hline $\begin{array}{c}Q \\
(\text { HCG-D) }\end{array}$ & 8.4 & 11.4 & 13.7 & 15.8 & 17.7 & 19.5 \\
\hline $\begin{array}{c}Q \\
\text { (MCG-E) }\end{array}$ & 10.4 & 15.8 & 20.9 & 26.0 & 31.0 & 36.0 \\
\hline $\begin{array}{c}Q \\
(\mathrm{COMB})\end{array}$ & 8.8 & 12.2 & 15.0 & 17.6 & 20.1 & 22.6 \\
\hline
\end{tabular}

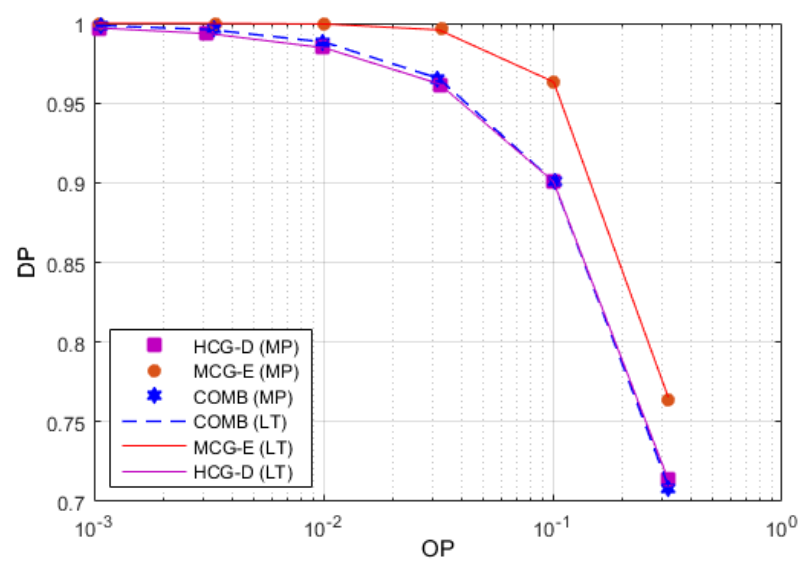

Hình 6. Khả năng giải mã của các nút nghe lén (DP) vẽ theo xác suất dì̀ng $(O P)$ khi $M=3, K=2$,

$$
N=3, \kappa_{\mathrm{D}}=\kappa_{\mathrm{E}}=0.5 \text { và } \kappa_{\mathrm{P}}=0.1
$$

Hình 6 mô tả sự đánh đổi giữa xác suất dừng $(\mathrm{OP})$ và xác suất giải mã (DP) của các giao thức khi $M=3$, $K=2, N=3, \kappa_{\mathrm{D}}=\kappa_{\mathrm{E}}=0.5$ và $\kappa_{\mathrm{P}}=0.1$. Trong mô phỏng này, chúng tôi cố định các giá trị của $\mathrm{OP}$ và sử dụng các công thức tính $\mathrm{OP}$ của các giao thức để tìm ra các giá trị $Q$ tương ứng (xem Bảng 1). Sau đó, các kết quả mô phỏng và lý thuyết của $\mathrm{DP}$ và $\mathrm{OP}$ sẽ được thực hiện, sử dụng các thông số vừa được thiết lập. Quan sát hình vẽ, ta thấy rằng có một sự đánh đổi giữa hiệu năng bảo mật và hiệu năng dừng của các mô hình. Cụ thể, để đạt được một giá trị xác suất dừng (OP) thấp thì giá trị DP lại lớn và ngược lại. Trong hình vẽ này, ta cũng thấy một điều thú vị là ở cùng giá trị của $\mathrm{OP}$ thì giá trị $\mathrm{DP}$ của mô hình MCG-E là lớn nhất, trong khi giá trị DP trong các mô hình HCG-D và $\mathrm{COMB}$ là gần như xấp xỉ. Điều đó cho thấy rằng mô hình MCG-E có hiệu năng thấp hơn khi so với hai mô hình còn lại.

\section{KẾT LUẬN}

Trong bài báo nghiên cứu bảo mật lớp vật lý trong mạng chuyển tiếp vô tuyến nhận thức dạng nền này, Chúng tôi đã đề xuất ba phương pháp lựa chọn nút chuyển tiếp để cải thiện hiệu năng của mạng người dùng thứ cấp thông qua tham số là xác suất dừng $(\mathrm{OP})$ và khả năng giải mã của các nút nghe lén (DP). Các biểu thức dạng đóng chính xác của $\mathrm{OP}$ và $\mathrm{DP}$ đã được đưa ra và được kiểm chứng bởi các mô phỏng Monter Carlo. Các kết quả cho thấy rằng mô hình HCG-D đạt được hiệu năng OP tốt nhất, mô hình MCG-E đạt được hiệu năng DP tốt nhất. Tuy nhiên, khi quan tâm đến sự đánh đổi giữa $\mathrm{OP}$ và $\mathrm{DP}$, hai mô hình $\mathrm{HCG}-\mathrm{D}$ và $\mathrm{COMB}$ đạt được hiệu quả tốt hơn.

\section{LỜI CẢM ƠN}

Nghiên cứu này được tài trợ bởi Học viện Công nghệ Bưu chính Viễn thông với đề tài mã số X_HV2017-RD ĐT2.

\section{TÀI LIỆU THAM KHẢO}

[1] J. MitolA, G. Q. J. MAGUIRE, "Cognitive Radio: Making Software Radios More Personal," IEEE Pers. Commun., vol. 6, no. 4, pp. 13 - 18, 1999.

[2] H. N. VU, T. D. TRAN, H. Y. KONG, "An Optimal Cooperative Spectrum Sensing Method in Cognitive 
Radio Network over Rayleigh Fading Channel," Proc. of IFIP Wireless Days, Niagara Falls, Canada, pp. 1 - 5, Oct. 2011.

[3] T. T. TRUC, H. Y. KONG, "Block-time of Arrival/Leaving Estimation to Enhance Local Spectrum Sensing under the Practical Traffic of Primary User," Journal of Communications and Networks, vol. 15, no. 5 , pp. $514-526$, Oct. 2013.

[4] T. Q. DUONG, V. N. Q. BAO, H. J. ZEPERNICK, "Exact Outage Probability of Cognitive AF Relaying with Underlay Spectrum Sharing," IET Electronics Letters, vol.47, no.17, pp. 1001 - 1002, Aug. 2011.

[5] P. N. SON, H. Y. KONG, "Exact Outage Analysis of Energy Harvesting Underlay Cooperative Cognitive Networks," IEICE Trans. on Commun., vol. E98-B, no. 4, pp. 661 - 672, Apr. 2015.

[6] T. T. DUY, G. C. ALEXANDROPOUlOS, T. T. VU, N.-S. VO, T. Q. DUONG, "Outage Performance of Cognitive Cooperative Networks with Relay Selection over Double-Rayleigh Fading Channels," IET Communications, vol. 10, no. 1, pp. 57 - 64, Jan. 2016.

[7] A. D. WYNER, “The Wire-tap Channel," In: AT\&T Bell Labs. Tech. J., vol. 54, no. 8, pp. 1355 - 1387, Oct. 1975.

[8] P. K. GOPALA, L. LAI, H. E. GAMAL, "On the Secrecy Capacity of Fading Channels," IEEE Trans. Inf. Theory, vol. 54, no. 10, pp. 4687 - 4698, Oct. 2008.

[9] I. KRIKIDIS, “Opportunistic Relay Selection for Cooperative Networks with Secrecy Constraints," IET Communications, vol. 4, no. 15, pp. 1787 - 1791, Oct. 2010 .

[10] L. SUN, T. ZHANG, Y. LI, H. NIU, "Performance Study of Two-Hop Amplify-and-Forward Systems With Untrustworthy Relay Nodes," IEEE Trans. Veh. Tech., vol. 61, no. 8, pp. $3801-3807$, Oct. 2012.

[11] Y. LIU, L. WANG, T. T. DUY, M. ELKASHLAN, TRUNG Q. DUONG, "Relay Selection for Security Enhancement in Cognitive Relay Networks," IEEE Wireless Commun. Lett., vol. 4, no. 1, pp. 46 - 49, Feb. 2015.

[12] D.-B. HA, TUNG T. VU, T. T. DUY, V. N. Q. BAO, "Secure Cognitive Reactive Decode-and-Forward Relay Networks: With and Without Eavesdropper," Wireless
Personal Communications, vol. 85, no. 4, pp. 2619 2641, Dec. 2015.

[13] Y. ZOU, B. CHAMPAGNE, W. P. ZHU, L. HANZO, "Relay-Selection Improves the Security-Reliability Trade-Off in Cognitive Radio Systems," IEEE Trans. on Commun., vol. 63, no. 1, pp. 215 - 228, Jan. 2015.

[14] M. MATTHAIOU, A. PAPADOGIANNIS, “Two-Way Relaying Under the Presence of Relay Transceiver Hardware Impairments," IEEE Commun. Lett., vol. 17, no. 6, pp. 1136--1139, Jun. 2013.

[15] E. BJORNSON, M. MATTHAIOU, M. DEBBAH, "New Look at Dual-hop Relaying: Performance Limits with Hardware Impairments," IEEE Trans. Commun., vol. 61, no. 11, pp. 4512 - 4525, Nov. 2013.

[16] D. T. HUNG, T. T. DUY, D. Q. TRINH, V. N. Q. BAO, T. HANH, "Impact of Hardware Impairments on Secrecy Performance of Multi-hop Relay Networks in Presence of Multiple Eavesdroppers," Proc. of NICS2016, Danang city, Viet Nam, pp. 113 - 118, Sep. 2016.

[17] P. T. D. NGOC, T. T. DUY, V. N. Q. BAO, N. L. NHAT, "Security-Reliability Analysis for Underlay Cognitive Radio Networks with Relay Selection Methods under Impact of Hardware Noises," Proc. of ATC2016, Hanoi, Viet Nam, pp. 174 - 179, Otc. 2016.

[18] P. T. D. NGOC, T. T. DUY, V. N. Q. BAO, H. V. KHUONG, "Transmit Antenna Selection Protocols in Random Cognitive Networks under Impact of Hardware Impairments," Proc. of NICS2016, Danang city, Viet Nam, pp. 38 - 43, Sep. 2016.

[19] P. M. QUANG, T. T. DUY AND V. N. Q. BAO, "Energy Harvesting-based Spectrum Access Model in Overlay Cognitive Radio," Proc. of ATC2015, Ho Chi Minh city, Viet Nam, pp. 231 - 236, Oct. 2015.

[20] T. T. DUY AND H. Y. KONG, "Performance Analysis of Incremental Amplify-and-Forward Relaying Protocols with Nth Best Partial Relay Selection under Interference Constraint," Wireless Personal Communications, vol. 71, no. 4, pp. 2741 - 2757, Aug. 2013.

Nhận bài ngày: 10/11/2016 


\section{SƠ LƯợC VỀ TÁC GIẢ}

\section{PHẠM THỊ ĐAN NGỌC}

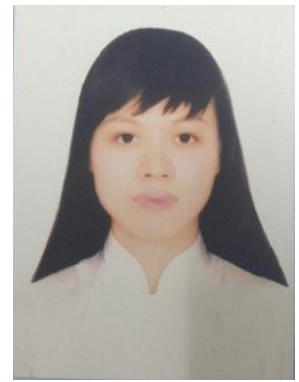

Sinh năm 1985.

Tốt nghiệp Thạc sĩ ngành vô tuyến tại Học viện Công nghệ Bưu chính Viễn thông cơ sở TP. HCM năm 2013.

Hiện công tác tại Học viện Công nghệ Bưu chính Viễn thông cơ sở

TP. HCM.

Hướng nghiên cứu : thông tin vô tuyến, vô tuyến nhận thức và khiếm khuyết phần cứng.

Email:ngocptd@ptithcm.edu.vn

\section{TRÀNN TRUNG DUY}

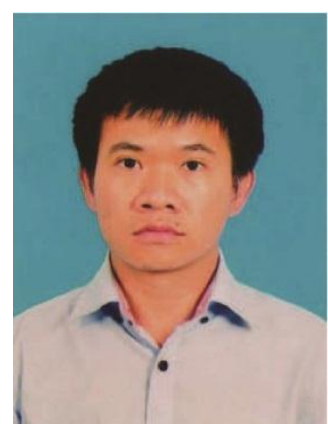

Sinh năm 1984.

Tốt nghiệp Tiến sĩ chuyên ngành vô tuyến tại ĐH Ulsan, Hàn Quốc năm 2013.

Hiện là giảng viên Khoa Viễn Thông 2, Học viện Công nghệ Bưu chính Viễn thông cơ sở TP. HCM.

Hướng nghiên cứu: khiếm khuyết phần cứng, bảo mật lớp vật lý và thu hoạch năng lượng vô tuyến.

Email: trantrungduy@ptithcm.edu.vn

\section{VÕ NGUYẼ̃N QUỐC BẢO}

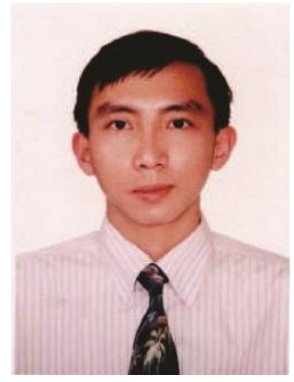

Năm sinh 1979.

Tốt nghiệp Tiến sĩ chuyên ngành vô tuyến tại ĐH Ulsan, Hàn Quốc năm 2010. Được phong PGS năm 2014.

Hiện công tác tại Học viện Công nghệ Bưu chính Viễn thông cơ sở

TP. HCM

Hướng nghiên cứu: vô tuyến nhận thức, truyền thông hợp tác, truyền song công, bảo mật lớp vật lý và thu thập năng lượng vô tuyến.

Email : baovnq@ptithcm.edu.vn

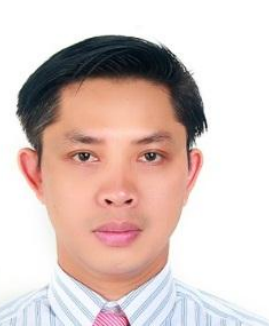

\section{HỒ VĂN KHƯƠNG}

Năm sinh 1978.

Nhận bằng Kỹ sư và Thạc sĩ trường $\mathrm{ĐH}$ Bách Khoa $\mathrm{TP}$ HCM năm 2003, bằng Tiến sĩ Đại học Ulsan, Hàn Quốc năm 2007.

Hiện công tác tại trường ĐH Bách Khoa, ĐH Quốc gia TP. HCM.

Hướng nghiên cứu: kỹ thuật điều chế và mã hóa, kỹ thuật phân tập, xử lý tín hiệu số, thu thập năng lượng, bảo mật lớp vật lý, và vô tuyến nhận thức.

Email: khuong.hovan@gmail.com 